\title{
PRESCRIBING SCALAR CURVATURES ON THE CONFORMAL CLASSES OF COMPLETE METRICS WITH NEGATIVE CURVATURE
}

\author{
ZHIREN JIN
}

\begin{abstract}
Let $\left(M^{n}, g\right)$ be a complete noncompact Riemannian manifold with the curvature bounded between two negative constants. Given a function $K$ on $M^{n}$, in terms of the behaviors of $K$ at infinite, we give a fairly complete answer to when the $K$ can be the scalar curvature function of a complete metric $g_{1}$ which is conformal to $g$.
\end{abstract}

\section{INTRODUCTION}

Let $\left(M^{n}, g\right)$ be a $n$-dimensional Riemannian manifold with a metric $g$, $n \geq 3$. An interesting problem in Riemannian geometry is to study if one can conformally deform the metric $g$ to a metric $g_{1}$ such that the scalar curvature of $g_{1}$ is a prescribed function $K$ on $M^{n}$. In the case that $M^{n}$ is compact and $K$ is a constant, this is just the famous Yamabe problem. The Yamabe problem had drawn a lot of attention from mathematicians because the equivalent PDE problem stands for a critical case that the standard analytic machinery can apply. The solution of the Yamabe problem was first claimed by Yamabe in 1960 [26]. In 1968, Trüdinger [25] found a serious gap in Yamabe's proof and filled the gap for the case that the metric has nonpositive scalar curvature. Later Aubin [2] showed the solution for the case $n \geq 6$ and $\left(M^{n}, g\right)$ is nonconformally flat. The remaining cases, which are the most difficult, were finally solved by Schoen [21]. Also see [15] for a unified proof in all cases. For recent progress of the general problem of prescribing scalar curvatures on a compact manifold, one may see [22] and [13].

In this paper, we consider the case that $\left(M^{n}, g\right)$ is complete noncompact. Since in this case, the metric $g$ is complete, we require the metric $g_{1}$ to also be complete. For the sake of clarity, we restate the problem.

Let $\left(M^{n}, g\right)$ be a complete noncompact Riemannian manifold, $n \geq 3$. Given a function $K$ on $\left(M^{n}, g\right)$, is there a complete metric $g_{1}$, which is conformal to $g$ and the scalar curvature of $g_{1}$ is $K$ ?

Received by the editors October 8, 1991.

1991 Mathematics Subject Classification. Primary 58E20; Secondary 35J50.

Key words and phrases. Open complete Riemannian manifolds, scalar curvature, pointwise conformal change of metrics, nonexistence of solutions of an elliptic equation, subsolution, supersolution, completeness.

Supported in part by NSF grant DMS-8610730. 
If we write $g_{1}=u^{4 /(n-2)} g$ for some $u>0$ on $M^{n}$, then the solvability of the problem is equivalent to the solvability of the following PDE problem.

$$
\left\{\begin{array}{l}
-\gamma_{n} \Delta_{g} u+S_{0} u=K u^{(n+2) /(n-2)} \text { on } M^{n}, \\
u^{4 /(n-2)} g \text { is complete on } M^{n}, \\
u>0 \text { on } M^{n}
\end{array}\right.
$$

where $\gamma_{n}=4(n-1) /(n-2), \Delta_{g}$ is the Laplace operator with respect to the metric $g$, and $S_{0}$ is the scalar curvature of $g$.

For problem (P), there have been various kinds of results. In 1982, Ni considered the case that $\left(M^{n}, g\right)$ is the standard Euclidean space. By studying radial symmetric solutions and using the super- and subsolution method, $\mathrm{Ni}$ [17] was able to show some existence and nonexistence results for problem (P) in this case. In an attempt to understand the Yamabe problem for complete noncompact manifolds, Aviles and McOwen [6] showed that a complete metric, with scalar curvature bounded above by a negative constant outside a compact set, can always be conformally deformed to a complete metric with negative constant scalar curvature. While in [12], we demonstrated counterexamples to show that the Yamabe problem for complete noncompact manifolds is not always solvable. For the general problem of prescribing scalar curvatures on a complete manifold with negative curvature (the problem was raised as an open problem in [13]), Aviles and McOwen [5] showed the following existence result (Theorem 4 in [5]).

Theorem. Suppose $M^{n}$ is complete, noncompact and simply connected. For some positive constants $A, B$, sectional curvature $\operatorname{Sec}(g)$ of $g$ satisfies $-A^{2} \leq$ $\operatorname{Sec}(g) \leq-B^{2}$. Let $r(x)$ be the distance of a point $x$ to a fixed point with respect to the metric $g$. Then problem $(\mathrm{P})$ is solvable if

(i) $A^{2} / B^{2} \leq(n-1)^{2} / n(n-2)$;

(ii) $-C_{2} \leq K(x) \leq-C_{1}$ for $r(x) \geq r_{0}$, where $C_{2}, C_{1}, r_{0}$ are some positive constants;

(iii) $\max _{M^{n}} K \leq C\left(r_{0}, A, B, n, C_{1}\right)\left(A^{2} / B^{2}-(n-1)^{2} / n(n-2)\right)$, where $C\left(r_{0}, A, B, n, C_{1}\right)$ is a constant.

Furthermore, if (ii) holds for $r_{0}=0$, then the conditions (i) and (iii) are not necessarly, and the solution is unique if $K \leq 0$ on $M^{n}$.

The goal of this paper is to continue to study the problem of prescribing scalar curvatures on complete noncompact manifolds with negative curvature. Our basic assumptions on the manifold $\left(M^{n}, g\right)$ are

$M^{n}$ is complete, noncompact and simply connected; the curvature of $g$ satisfies $\operatorname{Sec}(g) \leq-B^{2}, \operatorname{Ricc}(g) \geq-(n-1) A^{2}$ for some positive constants $A$, $B$.

Actually our results are true for weaker curvature assumptions. We will remark on this point at appropriate places. The results we get will give, in terms of the behaviors at infinity of the prescribed function $K$, a fairly complete answer to when $K$ can be the scalar curvature of a complete metric $g_{1}$ which is conformal to $g$.

Here are our main results. We assume the basic assumptions for all results. Furthermore, we assume $n \geq 4$ in the first theorem and $n \geq 3$ in the rest. For the results corresponding to Theorem 1 when $n=3$, see Theorem A and 
Theorem $\mathrm{B}$ in $\S 3$. The distance of a point $x$ to a fixed point with respect to the metric $g$ will be denoted by $r(x)$

The first result says that if $K$ is nonnegative near $\infty$, then problem (P) is not solvable.

Theorem $1(n \geq 4)$. If there is $r_{0}>0$, such that $K(x) \geq 0$ for $r(x) \geq r_{0}$. Then problem (P) has no solutions.

The second result says that if $K$ tends to negative infinity as $r \rightarrow \infty$, then problem $(\mathrm{P})$ is not solvable.

Theorem $2(n \geq 3)$. If there are positive constants $C, r_{0}, \sigma$, such that $K(x) \leq$ $-C e^{\sigma r(x)}$ for $r(x) \geq r_{0}$, then problem (P) has no solutions.

The third result says that problem $(\mathrm{P})$ is solvable for the "rest cases".

Theorem $3(n \geq 3)$. If $K \leq 0$ on $M^{n}$, and there are positive constants $C_{1}, C_{2}$, $\alpha, k, r_{0}$, such that $-C_{2} \leq K(x) \leq-C_{1} e^{-\alpha r(x)^{k}}$ for $r(x) \geq r_{0}$, then problem (P) has at least one solution.

Using the inhomogeneity of the equation in problem $(\mathrm{P})$, we see that Theorem 3 is a special case of the following more general result which we will prove.

Theorem $4(n \geq 3)$. Given positive constants $A, B, r_{0}, \alpha, k$, there is a constant $C\left(A, B, n, r_{0}, k, \alpha\right)>0$, such that if $\max _{M^{n}} K \leq C\left(A, B, n, r_{0}, k, \alpha\right)$, and for some positive constant $C_{2},-C_{2} \leq K(x) \leq-e^{-\alpha r(x)^{k}}$ for $r(x) \geq r_{0}$. Then problem (P) has at least one solution.

It is clear that Theorem 4 generalizes Aviles and McOwen's result.

From the analysis point of view, in the case that $\left(M^{n}, g\right)$ has negative curvature, the difficulties for solving (P) mainly arise from the noncompactness of the manifold $M^{n}$ and the generality of the metric. For nonexistence results, our idea is to look at the spherical average of the solution $u$ on the geodesic spheres. By studying the ordinary differential inequalities for the spherical average induced from the equation in problem $(\mathrm{P})$, we are able to prove that the metric $g_{1}=u^{4 /(n-2)} g$ is not complete for a solution $u$ of problem $(\mathrm{P})$, therefore showing a contradiction. Here one of the main technical difficulties is that the estimates of the volume element from the curvature assumptions only allow one control the first order derivatives of the volume element. This difficulty is overcome by an adoption of an idea used in the beautiful proofs of the unique continuation theorems appeared in [9] and [14] (see Remark 1 in $\S 3)$. For existence results, the method is the super-sub-solution method; in this case, a careful computation and scaling of the metric are needed for us to prove the present result.

When $n=2$, the problem of prescribing scalar curvatures has also been received extensive study. When $\left(M^{2}, g\right)$ is of negative curvature, the problem is greatly simplified by the Ahlfors-Schwarz lemma. For further detailed results when $n=2$, one might see $[4,5,7,16,18,19,20]$.

The paper is organized as follows. In $\S 2$, we present some results that will be needed in the proofs. In $\S 3$, we prove the nonexistence results. The existence results will be proved in $\S 4$.

Acknowledgment. The author would like to thank Professor Jerry Kazdan for helpful suggestions and Dr. S. H. Zhu for helpful discussions. This research 
was done while the author was visiting the Institute for Advanced Study during the 1990-1991 academic year.

\section{Preliminaries AND NOTATION}

In this section, we fix notation and present some known results which will be needed in the proofs.

Let $\operatorname{Sec}(g)$ be the sectional curvature of $g$, Ricc $(g)$ the Ricci curvature of $g$, and $S_{0}$ the scalar curvature of $g$.

Throughout the paper, we will assume that the manifold $\left(M^{n}, g\right)$ satisfies (we refer to these assumptions as basic assumptions).

$\left(M^{n}, g\right)$ is complete, noncompact and simply connected; for some positive constants $A, B, \operatorname{Sec}(g) \leq-B^{2}, \operatorname{Ricc}(g) \geq-(n-1) A^{2}$.

Fix a point, say $o$, in $M^{n}$, choose a normal coordinate center at $o$, denote the coordinate of points as $x$. By our assumptions on $\left(M^{n}, g\right), M^{n}$ is diffeomorphic to $R^{n}$ under this normal coordinate. If we identify $M^{n}$ with $R^{n}$ in this way, we have two metrics $g_{0}$ and $g$ on $R^{n}$ (or on $M^{n}$ ), $g$ is the original metric on $M^{n}, g_{0}$ is the Euclidean metric. Later on, when we refer to $R^{n}$ with the Euclidean metric, we mean $R^{n}$ (or $M^{n}$ ) with the metric $g_{0}$.

Let $r(x)$ be the distance of $x$ to $o$ with respect to $g$. Then using the polar coordinates of $x$, we can write the metric $g$ as

$$
g=g_{i j} d x^{i} \otimes d x^{j}=d r^{2}+r^{2} g_{\theta_{k} \theta_{l}} d \theta^{k} \otimes d \theta^{l} .
$$

Set $\sqrt{g}=\sqrt{\operatorname{det}\left(g_{i j}\right)_{1 \leq i, j \leq n}}, \sqrt{g}{ }_{\theta}=\sqrt{\operatorname{det}\left(g_{\left.\theta_{k} \theta_{l}\right)_{1 \leq k, l \leq n-1}}\right.}$.

We will denote the volume form of $g_{0}$ by $d x$. Denote the volume form on the unit sphere $S^{n-1}$ in the Euclidean space by $d \theta$. Then under the metric $g$, the volume form on the geodesic sphere $S_{r}=\{x \mid r(x)=r\}$ is $\sqrt{g}(r) d \theta$. Under the metric $g_{0}$, the volume form on $S_{r}$ is $r^{n-1} d \theta$.

The following result estimates $\sqrt{g}$ and $\sqrt{g}_{\theta}$.

Proposition 1. Let $\left(M^{n}, g\right)$ be a complete Riemannian manifold. $\operatorname{Ricc}(g) \geq$ $-(n-1) A^{2}, \operatorname{Sec}(g) \leq-B^{2}$. Then for $r>0$,

$$
\begin{gathered}
-n(n-1) A^{2} \leq S_{0} \leq-n(n-1) B^{2} ; \\
\left(\frac{\sinh B r}{B r}\right)^{n-1} \leq \sqrt{g_{\theta}}(r) \leq\left(\frac{\sinh A r}{A r}\right)^{n-1} ; \\
(n-1)\left(B \operatorname{coth} B r-\frac{1}{r}\right) \leq \frac{\partial \log \sqrt{g_{\theta}}}{\partial r} \leq(n-1)\left(A \operatorname{coth} A r-\frac{1}{r}\right) ; \\
(n-1) B \operatorname{coth} B r \leq \frac{\partial \log \sqrt{g}}{\partial r} \leq(n-1) A \operatorname{coth} A r ;
\end{gathered}
$$

Proof. (1) is trivial. (2), (3) are consequences of (4) if one notices that $r^{n-1} \sqrt{g_{\theta}}=\sqrt{g}$ and $\sqrt{g_{\theta}}(0)=1$.

For (4), since $\Delta_{g} r=\partial \log \sqrt{g} / \partial r$, we only have to prove that for $r>0$,

$$
(n-1) B \operatorname{coth} B r \leq \Delta_{g} r \leq(n-1) A \operatorname{coth} A r \text {. }
$$

The left-hand inequality follows from the comparison theorem for the Hessian (for example, see Theorem A on p. 19 in [11].) The right-hand side inequality 
follows from the argument presented in the proof of the Proposition 4.1 on $p$. 231 in [6] .

For notational convenience, we rewrite the equation in problem $(\mathrm{P})$. Since the equation is inhomogeneous about the unknown function $u$, it is clear that $(\mathrm{P})$ is equivalent to following problem. (We still denote it by $(\mathrm{P})$.)

$$
\left\{\begin{array}{l}
-\Delta_{g} u+S u=K u^{(n+2) /(n-2)} \text { on } M^{n}, \\
u^{4 /(n-2)} g \text { is complete on } M^{n} \\
u>0 \text { on } M^{n}
\end{array}\right.
$$

where $S=\gamma_{n}{ }^{-1} S_{0}=((n-2) / 4(n-1)) S_{0}$, and $\Delta_{g}$ is the Laplace operator with respect to the metric $g$.

Let $H^{n}(-1)$ be the hyperbolic space form, that is, $H^{n}(-1)$ is a complete, noncompact, simply connected manifold with constant sectional curvature -1 . $H_{\text {loc }}^{2}\left(M^{n}\right)=\left\{v \mid v, d v \in L_{\text {loc }}^{2}\left(M^{n}\right)\right\}$.

For the solvability of $(P)$, the following result will be needed in the proof of Theorem 4.

Proposition 2. Let $u^{+}, u_{-}$be two continuous functions in $H_{l o c}^{2}\left(M^{n}\right)$, such that

$$
\begin{array}{rlrl}
-\Delta_{g} u^{+}+S u^{+} & \geq K\left(u^{+}\right)^{(n+2) /(n-2)} & & \text { on } M^{n} \text { weakly; } \\
-\Delta_{g} u_{-}+S u_{-} & \leq K\left(u_{-}\right)^{(n+2)(n-2)} & & \text { on } M^{n} \text { weakly; } \\
u_{-} & \leq u^{+} \quad \text { on } M^{n} . &
\end{array}
$$

Then there is a $C^{2}$ solution $u$ to the equation in problem $(\mathbf{P})$ that satisfies $u_{-} \leq u \leq u^{+}$.

Proof. See the proof of Proposition 2.1 on p. 229 in [6]. In fact, in the case that both $u^{+}$and $u_{-}$are $C^{2}$, the result is well known. The observation needed in the nonsmooth case is that the maximum principles are still available in this case. Then the proof of the smooth case can be adopted without much change.

\section{NONEXISTENCE RESULTS}

In this section, under the basic assumptions about the manifold, we prove nonexistence results for problem $(\mathrm{P})$.

When $K \geq 0$ near $\infty$, we deal with $n=3$ and $n \geq 4$ separately. For $n \geq 4$, we can derive a better result at the present stage (Theorem 1 ). The case that $n=3$ will be treated in Theorems A and $\mathrm{B}$.

Theorem $1(n \geq 4)$. If there is $r_{0}>0$, such that $K(x) \geq 0$ for $r(x) \geq r_{0}$, then problem $(\mathrm{P})$ has no solutions.

Proof. We prove the result by contradiction. That is, if $u$ is a solution of problem (P), we will show that $u^{4 /(n-2)} g$ is not complete.

Without loss of generality (by multiplying $g$ by a suitable constant), we may assume

$$
A=1, \quad B<\min \left\{1,(n-1)^{2} / 16 n(n-2)\right\} .
$$


Then for $\nu=\left\{n(n-2) / 4(n-1)^{2}\right\} B^{2}$, it is easy to verify

$$
-\frac{1}{n-1}\left|S_{1}\right| \frac{1+\sqrt{1-4 \nu}}{2 \nu}+(n-1)(1-B)<0
$$

where $S_{1}=-\{n(n-2) / 4\} B^{2}=-\gamma_{n}^{-1} n(n-1) B^{2}$. Therefore we can choose a constant $0<\beta<1$ such that for $b=\left\{\beta^{2} /(n-1)^{2}\right\}\left|S_{1}\right|$, we have

$$
-\frac{\beta}{n-1}\left|S_{1}\right|\left(1+\frac{1-2 b+\sqrt{1-4 b}}{2 b}\right)+(n-1)(1-B)<0 \text {. }
$$

Define

$$
v(r)=\frac{1}{r^{n-1}} \int_{\partial B(r)} u \sqrt{g} d \theta
$$

and

$$
F(r)=\frac{1}{r^{n-1}} \int_{\partial B(r)} \frac{\partial u}{\partial r} \sqrt{g} d \theta / \frac{1}{r^{n-1}} \int_{\partial B(r)} u \sqrt{g} d \theta
$$

Then

$$
\begin{aligned}
v^{\prime}(r) & =\left(\frac{1}{r^{n-1}} \int_{\partial B(r)} u \sqrt{g} d \theta\right)^{\prime}=\left(\int_{S^{n-1}} u \sqrt{g_{\theta}} d \theta\right)^{\prime} \\
& =\frac{1}{r^{n-1}} \int_{\partial B(r)} \frac{\partial u}{\partial r} \sqrt{g} d \theta+\frac{1}{r^{n-1}} \int_{\partial B(r)} u \frac{\partial \log \sqrt{g}}{\partial r} \sqrt{g} d \theta .
\end{aligned}
$$

From the equation in $(P)$, we have

$$
\int_{B(r)}-\Delta_{g} u d \operatorname{vol}_{g}+\int_{B(r)} S u d \operatorname{vol}_{g}=\int_{B(r)} K u^{(n+2) /(n-2)} d \operatorname{vol}_{g} .
$$

Integrating by parts, we get

$$
\left(\int_{\partial B(r)} \frac{\partial u}{\partial r} \sqrt{g} d \theta\right)^{\prime}=\int_{\partial B(r)}\left(S u-K u^{(n+2) /(n-2)}\right) \sqrt{g} d \theta .
$$

A computation shows

$$
\begin{aligned}
F^{\prime}(r)= & -\frac{n-1}{r} F(r)-F(r)^{2}-\kappa(r) F(r) \\
& +\frac{1}{r^{n-1}} \int_{\partial B(r)}\left(S u-K u^{(n+2) /(n-2)}\right) \sqrt{g} d \theta / \frac{1}{r^{n-1}} \int_{\partial B(r)} u \sqrt{g} d \theta,
\end{aligned}
$$

where $\kappa(r)$ is defined as

$$
\kappa(r)=\frac{1}{r^{n-1}} \int_{\partial B(r)} u \frac{\partial \log \sqrt{g}}{\partial r} \sqrt{g} d \theta / \frac{1}{r^{n-1}} \int_{\partial B(r)} u \sqrt{g} d \theta .
$$

Since $-K \leq 0$ for $r \geq r_{0}$, and $S \leq-\gamma_{n}^{-1} n(n-1) B^{2}=S_{1}$, we have

$$
F^{\prime}(r) \leq-[(n-1) / r] F(r)-F^{2}(r)-\kappa(r) F(r)+S_{1} \text { for } r \geq r_{0} .
$$

We claim that there is $r_{1} \geq r_{0}$, such that

$$
F(r)<0 \text { for } r>r_{1} \text {. }
$$


In fact, by $(3)$, for $r>0$,

$$
(n-1)(B \operatorname{coth} B r-1 / r) \leq \kappa(r) .
$$

Hence there is $r_{2}$, such that for $r>r_{2}, \kappa(r)>0$. By (8), our claim follows if we can prove that there is a $r_{1}>r_{2}$, such that $F\left(r_{1}\right)<0$. If not, $F(r)>0$ for $r>r_{2}$, then from (8), we have

$$
F(r) \leq S_{1}\left(r-r_{2}\right)+F\left(r_{2}\right) \text { for } r>r_{2} .
$$

For $r$ large this contradicts the assumption that $F(r)>0$.

Now by $(8)$,

$$
F^{\prime}(r)+((n-1) / r+\kappa(r)) F(r) \leq S_{1} .
$$

Integrating (11), using (9), for $r_{3}>r_{1}$, we get

$$
F(r) \leq \frac{S_{1}}{r^{n-1}} \int_{r_{3}}^{r} t^{n-1} e^{-\int_{t}^{r} \kappa(\alpha) d \alpha} d t .
$$

But from (3), it is easy to see that for $r>t$ (since $A=1$ in our case)

$$
e^{-\int_{t}^{r} \kappa(\alpha) d \alpha} \geq\left(\frac{\sinh t}{\sinh r}\right)^{n-1} \frac{r^{n-1}}{t^{n-1}} .
$$

Equations (12) and (13) imply

$$
F(r) \leq S_{1} \int_{r_{3}}^{r}\left(\frac{\sinh t}{\sinh r}\right)^{n-1} d t .
$$

Therefore

$$
\begin{aligned}
F(r) & \leq S_{1} \int_{r_{3}}^{r} \frac{\left(1-e^{-2 r_{3}}\right)^{n-1}}{e^{(n-1) r}} e^{(n-1) t} d t \\
& =\frac{S_{1}}{n-1}\left(1-e^{-2 r_{3}}\right)^{n-1}\left(1-e^{-(n-1)\left(r-r_{3}\right)}\right) .
\end{aligned}
$$

Then for the $\beta<1$ chosen at the beginning of the proof, there are $r_{3}, r_{4}$, such that for $r \geq r_{4}>r_{3}$,

$$
F(r) \leq \frac{\beta}{(n-1)} S_{1}
$$

Since $S_{1}<0$, from (8), we have

$$
F^{\prime}(r)+((n-1) / r+\kappa(r)) F(r) \leq-\left(\left|S_{1}\right|+\left[\beta^{2} /(n-1)^{2}\right] S_{1}^{2}\right)
$$

Now if we go through the proof of (14) once again, but replace $S_{1}$ by $-\left(\left|S_{1}\right|+\beta^{2} S_{1}^{2} /(n-1)^{2}\right)$, we see that there is an $r_{5}$ such that for $r>r_{5}$,

$$
F(r) \leq-\frac{\beta}{n-1}\left(\left|S_{1}\right|+\frac{\beta^{2}}{(n-1)^{2}} S_{1}^{2}\right) .
$$

In general, for the $b$ defined at the beginning of the proof, if

$$
F(r) \leq-\frac{\beta}{n-1}\left|S_{1}\right|\left(1+b b_{i}\right) \text { for some } b_{i}>0 \text { and } r>r_{i} \text {, }
$$

we can conclude that there is $r_{i+1}$, such that for $r>r_{i+1}$,

$$
F(r) \leq-\{\beta /(n-1)\}\left|S_{1}\right|\left(1+b\left(1+b b_{i}\right)^{2}\right) .
$$


Therefore we get a sequence $\left\{b_{i}\right\}_{0}^{\infty}$, which is defined by

$$
b_{i+1}=\left(1+b b_{i}\right)^{2} \text { for } i>1, b_{0}=1 \text {. }
$$

It is clear that the sequence $\left\{b_{i}\right\}$ is monotone increasing. Then there are two possibilities: (i) $\left\{b_{i}\right\}$ is bounded; (ii) $\left\{b_{i}\right\}$ is unbounded. In the following, we show that $u^{4 /(n-2)} g$ is not complete in both cases.

In case $(\mathrm{i})$, there exists a limit. Say $\lim _{i \rightarrow \infty} b_{i}=\lambda$.

Then $\lambda=(1+b \lambda)^{2}$. Since $\lambda>0$,

$$
\lambda=(1-2 b+\sqrt{1-4 b}) / 2 b^{2} .
$$

$\lambda$ is real only when $1>4 b$. If $\lambda$ is real, from (16) and (6), we get

$$
-\frac{\beta}{n-1}\left|S_{1}\right|\left(1+\frac{1-2 b+\sqrt{1-4 b}}{2 b}\right)=-\frac{\beta}{n-1}\left|S_{1}\right|(1+b \lambda) .
$$

We see that there are $\eta<-(n-1)(1-B)$, and $r_{\eta}$, such that

$$
F(r) \leq \eta \quad \text { for } r>r_{\eta} \text {. }
$$

Then for $r>r_{\eta}$,

$$
\frac{1}{r^{n-1}} \int_{\partial B(r)} \frac{\partial u}{\partial r} \sqrt{g} d \theta \leq \eta \frac{1}{r^{n-1}} \int_{\partial B(r)} u \sqrt{g} d \theta
$$

By (7),

$$
v^{\prime}(r) \leq(\eta+\kappa(r)) v(r) \text { for } r>r_{\eta} .
$$

Also from (3), we see that for any $\varepsilon>0$, there is $r_{\varepsilon}$, such that

$$
\kappa(r) \leq(n-1)+\varepsilon \text { for } r>r_{\varepsilon} \text {. }
$$

Then for $r_{6}=r_{\eta}+r_{\varepsilon}$,

$$
v^{\prime}(r) \leq(\eta+(n-1)+\varepsilon) v(r) \quad \text { for } r>r_{6} .
$$

Therefore for $r>r_{6}$, we get

$$
v(r) \leq v\left(r_{6}\right) e^{(\eta+(n-1)+\varepsilon)\left(r-r_{6}\right)}
$$

Combining this with the definition of $v(r)$,

$$
\int_{S^{n-1}} u(r, \theta) \sqrt{g_{\theta}} d \theta \leq C\left(r_{6}, \eta, u\right) e^{(\eta+(n-1)+\varepsilon) r} .
$$

But (2) implies

$$
\sqrt{g_{\theta}} \geq C\left(B, r_{6}\right) e^{(n-1) B r} \frac{1}{r^{n-1}} \quad \text { for } r>r_{6} .
$$

Finally we get

$$
\int_{S^{n-1}} u(r, \theta) d \theta \leq C\left(r_{6}, \eta, u, B\right) e^{(\eta+(n-1)(1-B)+\varepsilon) r}
$$

Since $\eta+(n-1)(1-B)<0$, we can choose $\varepsilon$ such that $\eta+(n-1)(1-B)+\varepsilon<0$.

Then since $2 /(n-2) \leq 1$ for $n \geq 4$, we have

$$
\begin{aligned}
\int_{R^{n}} u^{2 /(n-2)} d x & \leq C \int_{0}^{\infty} r^{n-1}\left(\int_{S^{n-1}} u(r, \theta) d \theta\right)^{2 /(n-2)} \\
& \leq C \int_{0}^{\infty} r^{n-1} e^{2(\eta+(n-1)(1-B)+\varepsilon) r /(n-2)}<\infty .
\end{aligned}
$$


That is,

$$
\int_{S^{n-1}}\left(\int_{0}^{\infty} r^{n-1} u^{2 /(n-2)}(r, \theta) d r\right) d \theta<\infty .
$$

Then for some $\theta_{0} \in S^{n-1}$,

$$
\int_{0}^{\infty} r^{n-1} u^{2 /(n-2)}\left(r, \theta_{0}\right) d r<\infty
$$

Therefore

$$
\int_{0}^{\infty} u^{2 /(n-2)}\left(r, \theta_{0}\right) d r<\infty
$$

That is, for the metric $u^{4 /(n-2)} g$, the curve $C=\left\{\left(r, \theta_{0}\right) \mid 0<r<\infty\right\}$ has finite length. Thus the metric $u^{4 /(n-2)} g$ is not complete.

For case (ii) where $\left\{b_{i}\right\}_{0}^{\infty}$ is unbounded, it is obvious that we can choose $\eta<-(n-1)(1-B)$ and $r_{\eta}$ such that (17) is true. Then the same proof given above concludes that the metric $u^{4 /(n-2)} g$ is also not complete. This completes the proof of Theorem 1 .

Remark 1. In the proof, we did not take the second order derivative of $v(r)$ directly because the terms

$$
\frac{1}{r^{n-1}} \int_{\partial B(r)} \frac{\partial u}{\partial r} \frac{\partial \log \sqrt{g}}{\partial r} \sqrt{g} d \theta ; \quad \frac{1}{r^{n-1}} \int_{\partial B(r)} u \frac{\partial^{2} \log \sqrt{g}}{\partial r^{2}} \sqrt{g} d \theta
$$

cannot be estimated directly by the assumptions on the curvature and the equation in problem $(\mathrm{P})$. This is the reason that we introduce the function $F(r)$. This idea has been used in [9] and [14] to study the unique continuation property for solutions of elliptic equations and systems. The same remark also applies to the proof of Theorem 2 .

Remark 2. If one checks the proof of Theorem 1 carefully, it is easy to see that the proof gets through if one has the following estimates which are weaker than the estimates obtained in Proposition 1:

(a) There is $r_{1}>0$, such that

$$
\frac{\partial \log \sqrt{g_{\theta}}}{\partial r} \geq 0 \text { for } r>r_{1}
$$

(b) There is a function $\varepsilon(r), \varepsilon(r) \longrightarrow 0$ as $r \longrightarrow \infty$, and $r_{1}>0$, such that

$$
\frac{\partial \log \sqrt{g_{\theta}}}{\partial r} \leq(n-1) A+\varepsilon(r) \text { for } r>r_{1},
$$

(c) There are positive constants $C, r_{1}$ and a function $\mu(r), \mu(r) \rightarrow 0$ as $r \rightarrow \infty$, such that

$$
\sqrt{g_{\theta}} \geq C e^{(n-1) B r+\mu(r) r} \quad \text { for } r>r_{1}
$$

Remark 3. There may exist a positive solution $u$ to the equation in problem (P) for some $K$ with $K \geq 0$ near $\infty$. By Theorem $1, u^{4 /(n-2)} g$ is not complete. 
Example 1. Let $\left(M^{n}, g\right)$ be the space form. That is, $\left(M^{n}, g\right)=H^{n}(-1)$ $=\left(D^{n}, g_{2}\right)$, where $D^{n}$ is the unit ball in the Euclidean space. Since $g_{2}=$ $4 /\left(1-|x|^{2}\right)^{2} d x^{2}, v=\left(2 /\left(1-|x|^{2}\right)\right)^{-(n-2) / 2}$ satisfies the corresponding equation with $K=0$. And $g_{1}=v^{4 /(n-2)} g_{2}=d x^{2}$ is not complete on $D^{n}$.

Remark 4. From the proof, we see that the only place where we require $n>3$ is to assure $2 /(n-2) \leq 1$ in (20). But (19) is true for $n \geq 3$.

When $n=3$, we can prove the following results

Theorem $\mathbf{A}(n=3)$. If there are positive constants $C, r_{0}, \mu$, such that

$$
K(x) \geq C r(x)^{-\mu} \text { for } r(x)>r_{0},
$$

then problem $(\mathrm{P})$ has no solutions.

Proof. We will use some conclusions obtained in the proof of Theorem 1 to prove the result.

First of all, from the proof of $(21)$, we only have to prove that for a solution $u$ of problem $(\mathrm{P})$

$$
\int_{R^{3}} u^{2} d x<\infty
$$

From the equation in $(P)$, we have

$$
-\left(\int_{\partial B(r)} \frac{\partial u}{\partial r} \sqrt{g} d \theta\right)^{\prime}+\int_{\partial B(r)} S u \sqrt{g} d \theta=\int_{\partial B(r)} K u^{5} \sqrt{g} d \theta
$$

Notice $S<0$ and (2), for $r>1$,

$$
C(B) r^{-2} e^{2 B r} \int_{\partial B(r)} K u^{5} r^{2} d \theta \leq-\left(\int_{\partial B(r)} \frac{\partial u}{\partial r} \sqrt{g} d \theta\right)^{\prime} .
$$

Then for any $\xi>0$,

$$
C(B) r^{\xi} \int_{\partial B(r)} K u^{5} r^{2} d \theta \leq-r^{\xi+2} e^{-2 B r}\left(\int_{\partial B(r)} \frac{\partial u}{\partial r} \sqrt{g} d \theta\right)^{\prime}
$$

By (9) and integration by parts, for $r>r_{1}>r_{0}$,

$$
\begin{aligned}
& C(B) \int_{r_{1}}^{r} t^{\xi} \int_{\partial B(t)} K u^{5} t^{2} d \theta d t \leq-\int_{r_{1}}^{r} t^{\xi+2} e^{-2 B t}\left(\int_{\partial B(t)} \frac{\partial u}{\partial r} \sqrt{g} d \theta\right)^{\prime} d t \\
& \leq-r^{\xi+2} e^{-2 B r}\left(\int_{\partial B(r)} \frac{\partial u}{\partial r} \sqrt{g} d \theta\right)-2 B \int_{r_{1}}^{r} t^{\xi+2} e^{-2 B t}\left(\int_{\partial B(t)} \frac{\partial u}{\partial r} \sqrt{g} d \theta\right) d t .
\end{aligned}
$$

Notice that (7) can be rewritten as

$$
v^{\prime}(r) r^{2}=\int_{\partial B(r)} \frac{\partial u}{\partial r} \sqrt{g} d \theta+r^{2} \kappa(r) v(r) .
$$


Then (18), $\eta+2(1-B)+\varepsilon<0$, (3), integration by parts, and $v(r)>0$ imply that for $r_{2}=r_{1}+1$,

$$
\begin{aligned}
C(B) & \int_{r_{2}}^{r} \int_{r_{1}}^{s} t^{\xi} \int_{\partial B(t)} K u^{5} t^{2} d \theta d t d s \\
\leq & -\int_{r_{2}}^{r} s^{\xi+4} e^{-2 B s}\left(v^{\prime}(s)-\kappa(s) v(s)\right) d s \\
& -2 B \int_{r_{2}}^{r} \int_{r_{1}}^{s} t^{\xi+4} e^{-2 B t}\left(v^{\prime}(t)-\kappa(t) v(t)\right) d t d s \\
\leq & C_{1}\left(A, B, r_{1}, \xi\right) r+C_{2}\left(A, B, r_{1}, \xi\right) \text { for some constants } C_{1}, C_{2} .
\end{aligned}
$$

Now by Hölder inequality and $(22)$, we have

$$
C t^{\xi-\mu-3}\left(\int_{\partial B(t)} u^{2} t^{2} d \theta\right)^{5 / 2} \leq t^{\xi} \int_{\partial B(t)} K u^{5} t^{2} d \theta \text { for } t>r_{0}
$$

and

$$
\begin{aligned}
& \int_{r_{2}}^{r}\left(\int_{B(s) \backslash B\left(r_{1}\right)} u^{2} d x\right)^{5 / 2} d s \\
& \quad \leq \int_{r_{2}}^{r}\left(\int_{r_{1}}^{s} t^{\xi-\mu-3}\left(\int_{\partial B(t)} u^{2} t^{2} d \theta\right)^{5 / 2} d t\right)\left(\int_{r_{1}}^{s} t^{-2(\xi-\mu-3) / 3} d t\right)^{3 / 2} d s .
\end{aligned}
$$

If we choose $\xi=6+\mu$, then

$$
\left(\int_{r_{1}}^{s} t^{-2(\xi-\mu-3) / 3} d t\right)^{3 / 2}=\left(\int_{r_{1}}^{s} t^{-2} d t\right)^{3 / 2}<\infty
$$

Combining (24) with the above inequalities, we finally get that for $r>r_{2}$,

$$
\int_{r_{2}}^{r}\left(\int_{B(s) \backslash B\left(r_{1}\right)} u^{2} d x\right)^{5 / 2} d s \leq C_{1}\left(A, B, r_{1}, \mu\right) r+C_{2}\left(A, B, r_{1}, \mu\right) \text {. }
$$

Now it is clear that $(23)$ is true.

We can do better for the hyperbolic space form. The argument in the following result actually works for all dimensions $n \geq 3$.

Theorem B. If $\left(M^{3}, g\right)=H^{3}(-1)$ and there is a constant $r_{0}>0$ such that $K(x) \geq 0$ for $r(x)>r_{0}$, then problem (P) has no solutions.

Proof. This result is basically a consequence of Theorem 5.1 in [23]. Let $u$ be a solution of $(\mathrm{P})$. By assumption, $\left(M^{3}, g\right)=H^{3}(-1)=\left(D^{3}, g_{2}\right)=$ hyperbolic space form, where $D^{3}$ is the 3-dimensional unit ball. Denote Euclidean coordinates on $D^{3}$ by $x$ and $v=\left(2 /\left(1-|x|^{2}\right)\right)^{1 / 2}$. Then $g_{2}=v^{4} d x^{2}$. Now $g_{1}=u^{4} g_{2}=(u v)^{4} d x^{2}$ is complete and the scalar curvature of $g_{1}$ is $K$. Therefore

$$
\begin{aligned}
& -\gamma_{3} \Delta(u v)=K(u v)^{5} \text { on } D^{3} \\
& (u v)^{4} d x^{2} \text { is complete on } D^{3}
\end{aligned}
$$

where $\Delta$ is the Laplace operator of the standard Euclidean metric. 
Let $\phi$ be the first eigenfunction of the following Dirichlet problem.

$$
\begin{aligned}
-\Delta \phi & =\lambda_{1} \phi & & \text { on } D^{3} ; \\
\phi & =0 & & \text { on } \partial D^{3} .
\end{aligned}
$$

Then we may assume $\phi>0$ in $D^{3}$. For a large constant $C$, look at the metric $g_{3}=(u v+C \phi)^{4} d x^{2}$ on $D^{3}$. Since $\phi>0$ and $(u v)^{4} d x^{2}$ is complete on $D^{3}$, $g_{3}$ is complete on $D^{3}$. Furthermore, the scalar curvature of $g_{3}$ is

$$
-\gamma_{3}(\Delta(u v+C \phi))(u v+C \phi)^{-5}=\left(K(u v)^{5}+\lambda_{1} C \gamma_{3} \phi\right)(u v+C \phi)^{-5} .
$$

Since $\lambda_{1}>0$ and $K(x) \geq 0$ for $|x|>1-\delta$ with some $0<\delta<1$, it is clear that we can choose a large $C$ such that the scalar curvature of $g_{3}$ is nonnegative. Also since $D^{3}$ with the Euclidean metric $d x^{2}$ is conformal to a half-unit sphere $S_{+}^{3}$ with the standard metric $g_{4}$, we have a metric $g_{3}$ which is complete, conformal to $g_{4}$ on the half-sphere, and has nonnegative scalar curvature. By Theorem 5.1 in [23], this kind of metric does not exist. That is, (P) has no solutions.

Now we consider the case that $K$ tends to $-\infty$ as $r \longrightarrow \infty$.

Theorem $2(n \geq 3)$. If there are $\sigma>0, r_{0}>0, C>0$ such that

$$
K(x) \leq-C e^{\sigma r(x)} \text { for } r(x) \geq r_{0},
$$

then problem $(\mathrm{P})$ has no solutions.

Proof. As in proof of Theorem 1, we will prove that any positive solution $u$ to the equation in problem (P) will not make $u^{4 /(n-2)} g$ complete.

First of all, choose a constant $q$ such that $q>n /(n-2)$ and for $p=$ $1+4 /(n-2) q$

$$
\sigma-2(n-1)(p-1)(A-B)>0 .
$$

From (26), we can choose a constant $\mu<0$ such that

$$
\sigma-(n-1)(p-1)(A-B)+(p-1) \mu>0
$$

and

$$
\mu+(n-1)(A-B)<0 .
$$

Now define

$$
h(r)=\frac{1}{r^{n-1}} \int_{\partial B(r)} u^{q} \sqrt{g} d \theta
$$

and

$$
G(r)=\int_{\partial B(r)} u^{q-1} \frac{\partial u}{\partial r} \sqrt{g} d \theta
$$

Then it is easy to compute

$$
h^{\prime}(r)=q\left(1 / r^{n-1}\right) G(r)+\kappa_{q}(r) h(r)
$$

where $\kappa_{q}(r)$ is defined as

$$
\kappa_{q}(r)=\frac{1}{r^{n-1}} \int_{\partial B(r)} u^{q} \frac{\partial \log \sqrt{g_{\theta}}}{\partial r} \sqrt{g} d \theta / \frac{1}{r^{n-1}} \int_{\partial B(r)} u^{q} \sqrt{g} d \theta .
$$


PRESCRIBING SCALAR CURVATURES

797

Multiply $u^{q-1}$ on the both sides of the equation in the problem (P) and integrate by parts to get

$$
\begin{aligned}
-\int_{\partial B(r)} & u^{q-1} \frac{\partial u}{\partial r} \sqrt{g} d \theta+\int_{B(r)}(q-1) u^{q-2}|\nabla u|^{2} d \operatorname{vol}_{g}+\int_{B(r)} S u^{q} d \operatorname{vol}_{g} \\
= & \int_{B(r)} K u^{q p} d \operatorname{vol}_{g} .
\end{aligned}
$$

Therefore

$$
G^{\prime}(r)=\int_{\partial B(r)}(q-1) u^{q-2}|\nabla u|^{2} \sqrt{g} d \theta+\int_{\partial B(r)}\left(S u^{q}-K u^{q p}\right) \sqrt{g} d \theta .
$$

Set $S_{2}=-\gamma_{n}^{-1} n(n-1) A^{2}=-[n(n-2) / 4] A^{2}$, and for $r \geq r_{0}$,

$$
K_{1}(r)=\left\{\frac{1}{r^{n-1}} \int_{\partial B(r)}|K|^{-1 /(p-1)} \sqrt{g} d \theta\right\}^{-1} .
$$

Then for $r \geq r_{0}$, by the Holder inequality,

$$
\left(\frac{1}{r^{n-1}} \int_{\partial B(r)} u^{q} \sqrt{g} d \theta\right)^{p} \leq\left(\frac{1}{r^{n-1}} \int_{\partial B(r)}|K| u^{q p} \sqrt{g} d \theta\right) K_{1}^{-(p-1)} .
$$

Notice $K<0$ for $r>r_{0}$ and $q>1$, (30) implies

$$
G^{\prime}(r) / r^{n-1} \geq S_{2} h(r)+K_{1}^{p-1} h(r)^{p} .
$$

By (25) and (2), for $r \geq r_{0}$,

$$
K_{1} \geq C\left(r_{0}, A\right) r^{n-1} e^{-(n-1) A r+\sigma r /(p-1)} .
$$

Combing this with (31), for $r \geq r_{0}$, gives

$$
G^{\prime}(r) \geq r^{n-1}\left(S_{2} h+C r^{(n-1)(p-1)} e^{\sigma r-(n-1)(p-1) A r} h^{p}\right) .
$$

Rewrite (29) as

$$
\left(e^{-\int_{r_{0}}^{r} \kappa_{q}(t) d t} h(r)\right)^{\prime}=\frac{q}{r^{n-1}} e^{-\int_{r_{0}}^{r} \kappa_{q}(t) d t} G(r) .
$$

Then from (32), we have

$$
\begin{aligned}
& \left(r^{n-1} e^{\int_{r_{0}}^{r} \kappa_{q}(t) d t}\left(e^{-\int_{r_{0}}^{r} \kappa_{q}(t) d t} h(r)\right)^{\prime}\right)^{\prime} \\
& \quad \geq r^{n-1}\left(S_{2} h+C r^{(n-1)(p-1)} e^{\sigma r-(n-1)(p-1) A r} h^{p}\right) .
\end{aligned}
$$

Define

$$
w(r)=e^{-\int_{r_{0}}^{r} \kappa_{q}(t) d t} h(r) .
$$

Then (33) becomes

$$
\begin{aligned}
& \left(r^{n-1} e^{\int_{r_{0}}^{r} \kappa_{q}(t) d t} w^{\prime}(r)\right)^{\prime} \\
& \quad \geq q r^{n-1}\left(S_{2} w+C r^{(n-1)(p-1)} e^{\sigma r-(n-1)(p-1) A r+(p-1) \int_{r_{0}}^{r} \kappa_{q}(t) d t} w^{p}\right) e^{\int_{r_{0}}^{r} \kappa_{q}(t) d t} .
\end{aligned}
$$


That is,

$$
\begin{aligned}
w^{\prime \prime} & +\left(\frac{n-1}{r}+\kappa_{q}(r)\right) w^{\prime} \\
& \geq q S_{2} w+C r^{(n-1)(p-1)} e^{\sigma r-(n-1)(p-1) A r+(p-1) \int_{r_{0}}^{r} \kappa_{q}(t) d t} w^{p}
\end{aligned}
$$

Now (3) implies that for $r \geq r_{0}$,

$$
e^{(p-1) \int_{r_{0}}^{r} \kappa_{q}(t) d t} \geq \frac{C\left(r_{0}, n, B\right)}{r^{(n-1)(p-1)}} e^{(n-1)(p-1) B r} .
$$

Then

$$
w^{\prime \prime}+\left(\frac{n-1}{r}+\kappa_{q}(r)\right) w^{\prime} \geq q S_{2} w+C e^{\sigma r-(n-1)(p-1)(A-B) r} w^{p} .
$$

We will prove that the function $w$ approaches zero fast enough to conclude the metric $u^{4 /(n-2)} g$ is not complete.

Set

$$
y(r)=\xi e^{\mu r}
$$

where the constant $\xi$ will be specified in a moment and $\mu$ is determined by (27) and (28).

Then

$$
\begin{aligned}
y^{\prime \prime} & +\left(\frac{n-1}{r}+\kappa_{q}(r)\right) y^{\prime}-q S_{2} y-C e^{\sigma r-(n-1)(p-1)(A-B) r} y^{p} \\
& =y(r)\left\{\mu^{2}+\left(\frac{n-1}{r}+\kappa_{q}(r)\right) \mu-q S_{2}-C e^{\sigma r-(n-1)(p-1)(A-B) r+(p-1) \mu r} \xi^{p-1}\right\} .
\end{aligned}
$$

Therefore by (27) and (3), there is $r_{1}>1$, such that for arbitrary $\xi>1$, when $r>r_{1}$,

$$
y^{\prime \prime}+\left(\frac{n-1}{r}+\kappa_{q}(r)\right) y^{\prime} \leq q S_{2} y+C e^{\sigma r-(n-1)(p-1)(A-B) r} y^{p} .
$$

For the constant $C$ in $(38)$, choose $\xi$ large enough such that

$$
w\left(r_{1}\right)-\xi e^{\mu r_{1}}<0
$$

and

$$
q S_{2}+p C \xi^{p-1}>0
$$

Set $z(r)=w(r)-y(r)$. From (36) and (38), we have that for $r>r_{1}$,

$$
z^{\prime \prime}+\left(\frac{n-1}{r}+\kappa_{q}(r)\right) z^{\prime} \geq\left(q S_{2}+C e^{\sigma r-(n-1)(p-1)(A-B) r} p \rho(r)^{p-1}\right) z(r),
$$

where either $w(r) \leq \rho(r) \leq y(r)$ or $y(r) \leq \rho(r) \leq w(r)$.

Now we claim that $z(r)$ cannot achieve a local positive maximum on $\left(r_{1}, \infty\right)$. In fact if $z(r)$ does achieve a local positive maximum at $r_{3}>r_{1}$, then there are $r_{4}<r_{5} \leq \infty$ such that $r_{4}<r_{3}<r_{5}$ and $z(r) \geq 0$ on $\left(r_{4}, r_{5}\right)$. But $z(r) \geq 0$ implies $w(r) \geq y(r)=\xi e^{\mu r}$ and then $\rho(r) \geq \xi e^{\mu r}$. Therefore (40), (41), and $r_{1}>1$ imply

$$
\begin{aligned}
z^{\prime \prime}+ & \left(\frac{n-1}{r}+\kappa_{q}(r)\right) z^{\prime} \geq\left(q S_{2}+C e^{\sigma r-(n-1)(p-1)(A-B) r} p \rho(r)^{p-1}\right) z(r) \\
& \geq\left(q S_{2}+C p \xi^{p-1} e^{\sigma r-(n-1)(p-1)(A-B) r+(p-1) \mu r}\right) z(r) \geq 0 \text { on }\left(r_{4}, r_{5}\right) .
\end{aligned}
$$


But at $r_{3}, z\left(r_{3}\right)>0, z^{\prime}\left(r_{3}\right)=0, z^{\prime \prime}\left(r_{3}\right) \leq 0$. Thus we have a contradiction from (42). That is, our claim is true.

Observing (39), we see that there are only two possibilities:

(i) $z(r) \leq 0$ on $\left(r_{1}, \infty\right)$;

(ii) for some $r_{6}>r_{1}, z(r)>0, z^{\prime}(r) \geq 0$ on $\left(r_{6}, \infty\right)$.

We will prove that $u^{4 /(n-2)} g$ is not complete if (i) is true and (ii) will not happpen. If (i) holds,

$$
w(r) \leq \xi e^{\mu r} \text { for } r \geq r_{1} .
$$

Combing this with (34) and the definition of $h(r)$, we have

$$
\int_{S^{n-1}} u^{q}(r, \theta) \sqrt{g_{\theta}} d \theta \leq \xi e^{\mu r+\int_{r_{0}}^{r} \kappa_{q}(t) d t} \text { for } r \geq r_{1} .
$$

Then by (2),

$$
\int_{S^{n-1}} u^{q}(r, \theta) d \theta \leq \xi e^{\mu r+\int_{r_{0}}^{r} \kappa_{q}(t) d t} \frac{(B r)^{n-1}}{(\sinh B r)^{n-1}} \text { for } r \geq r_{1} .
$$

Using (3), we get

$$
\int_{S^{n-1}} u^{q}(r, \theta) d \theta \leq C\left(A, B, r_{1}, n\right) e^{\mu r+(n-1)(A-B) r} \quad \text { for } r \geq r_{1} .
$$

From (28) and noting that $q>2 /(n-2)$, we can proceed as in the proof of (21) to conclude that the metric $u^{4 /(n-2)} g$ is not complete.

If (ii) holds, pick up $r_{7}>r_{6}$. Then $z_{1}(r)=z(r) / z\left(r_{7}\right)$ satisfies $z_{1}(r)>1$, $z_{1}^{\prime}(r) \geq 0$ for $r>r_{7}$, and (41) implies

$$
\begin{aligned}
z_{1}^{\prime \prime}+ & \left((n-1) / r+\kappa_{q}(r)\right) z_{1}^{\prime} \\
& \geq\left(q S_{2}+C\left(A, B, \xi, n, r_{7}\right) e^{\sigma r-(n-1)(p-1)(A-B) r+(p-1) \mu r}\right) z_{1}(r) \quad \text { for } r>r_{7} .
\end{aligned}
$$

Here we used the fact that $w(r) \geq y(r)$. Then $\rho(r) \geq y(r)$ for $r>r_{1}$.

Denote $\gamma=\frac{1}{2}(\sigma-(n-1)(p-1)(A-B)+(p-1) \mu)$. Then there is $r_{8}>r_{7}$ such that for $r_{8}>r_{7}$,

$$
\left.z_{1}^{\prime \prime}+((n-1) / r)+\kappa_{q}(r)\right) z_{1}^{\prime} \geq C e^{\gamma r} z_{1}(r) .
$$

Since $z_{1}^{\prime}\left(r_{8}\right) \geq 0$, we get

$$
z_{1}^{\prime}(r) \geq C \int_{r_{8}}^{r} \frac{s^{n-1}}{r^{n-1}} e^{-\int_{s}^{r} \kappa_{q}(t) d t} e^{\gamma s} z_{1}(s) d s .
$$

Combing (44) with (3), we have

$$
z_{1}^{\prime}(r) \geq \frac{C}{e^{(n-1) A r}} \int_{r_{8}}^{r} e^{(n-1) A s+\gamma s} z_{1}(s) d s \text { for } r>r_{8} .
$$

Since $z_{1}(r) \geq 1$ for $r>r_{7}$, there is $r_{9}>r_{8}$ such that

$$
z_{1}^{\prime}(r) \geq \frac{C}{e^{(n-1) A r}} \frac{e^{(n-1) A r+\gamma r}}{(n-1) A+\gamma}\left(1-e^{((n-1) A+\gamma)\left(r_{8}-r\right)}\right) \geq C e^{\gamma r} \quad \text { for } r>r_{9}>r_{8} .
$$

That is $w^{\prime}(r)-y^{\prime}(r) \geq C e^{\gamma r}$ for $r>r_{9}$. Since $\mu<0, \gamma>0$, there is $r_{10}$ such that

$$
w^{\prime}(r) \geq C e^{\gamma r} \text { for } r \geq r_{10}
$$


Then for some $r_{11}>r_{10}$,

$$
w(r)>C e^{\gamma r} \text { for } r>r_{11} .
$$

Denote $\zeta=\frac{1}{2}(\sigma-(n-1)(p-1)(A-B))$. From (36) and (47), we have

$$
w^{\prime \prime}+\left((n-1) / r+\kappa_{q}(r)\right) w^{\prime} \geq C e^{\zeta r} w^{p} \quad \text { for } r>r_{12}>r_{11} .
$$

Notice that $\zeta>0,(46),(47)$ and (48) imply (similar to the proof of (45))

$$
\begin{aligned}
w^{\prime}(r) & \geq C \int_{r_{12}}^{r} \frac{1}{e^{(n-1) A r}} e^{(n-1) A s+\zeta s} w^{p} d s \\
& \geq C \int_{r_{12}}^{r} \frac{1}{e^{(n-1) A r}} e^{(n-1) A s+\zeta s+\gamma p s} d s \\
& \geq C e^{\gamma p r} \text { for } r>r_{13}>r_{12} .
\end{aligned}
$$

Thus for some $r_{14}>r_{13}$,

$$
w(r) \geq C e^{\gamma p r} \text { for } r>r_{14}
$$

Using (46), (48), and (49), go through the proof of (49) once again, we see that there is $r_{15}>r_{14}$ such that

$$
w(r) \geq C e^{\gamma p^{2} r} \text { for } r>r_{15} .
$$

Repeating the above procedure, in general, we can prove that for any integer $k$, there is $r_{k}$ such that

$$
w(r) \geq C e^{\gamma p^{k} r} \text { for } r>r_{k} .
$$

Denote $\nu=(2(n-1) A+\zeta) / \gamma p^{k}$. Since $p>1$, we can choose $k$ large enough such that

$$
2 p /(p+1+\nu)>1 .
$$

Now fixing one of these $k$ and combining (48) with (3), we have

$$
e^{(n-1) A r} w^{\prime}(r) \geq C \int_{r_{k}}^{r} e^{(n-1) A s+\zeta s} w^{p} d s .
$$

Define

$$
\psi(r)=\int_{r_{k}}^{r} e^{(n-1) A s+\zeta s} w^{p} d s .
$$

Then

$$
\psi^{\prime}(r)=e^{(n-1) A r+\zeta r} w^{p} .
$$

Using $\psi(r),(53)$ can be rewritten as

$$
\begin{aligned}
e^{2(n-1) A r+\zeta r} w^{p} w^{\prime}(r) & \geq C e^{(n-1) A r+\zeta r} w^{p} \int_{r_{k}}^{r} e^{(n-1) A s+\zeta s} w^{p} d s \\
& =C \psi(r) \psi^{\prime}(r) \quad \text { for } r>r_{k} .
\end{aligned}
$$

Then for $r>r_{k}$, by (51),

$$
C \psi(r) \psi^{\prime}(r) \leq w^{\prime}(r) w^{p}\left(e^{\gamma p^{k} r}\right)^{\nu} \leq C w^{\prime}(r) w^{p} w^{\nu}=C w^{\prime}(r) w^{p+\nu} .
$$


Integrating the above inequality from $r_{k}$ to $r$, we have

$$
w^{p+\nu+1}(r) \geq C \psi^{2}(r)+w^{p+\nu+1}\left(r_{k}\right)-C \psi^{2}\left(r_{k}\right) \text { for } r>r_{k} .
$$

Combining this with (51) and the definition of $\psi(r)$, we can choose $R_{k}>r_{k}$ such that

$$
w^{p+\nu+1}(r) \geq C \psi^{2}(r) \text { for } r>R_{k},
$$

i.e.,

$$
w^{p}(r) \geq C \psi^{2 p /(p+\nu+1)}(r) \quad \text { for } r>R_{k} .
$$

Therefore, by (54), we have

$$
\psi^{\prime}(r) \geq C e^{(n-1) A r+\zeta r} \psi^{2 p /(p+\nu+1)}(r) \quad \text { for } r>R_{k} .
$$

Finally, integrating the above inequality from $R_{k}$ to $r$, we get

$$
\begin{aligned}
& \frac{1}{1-2 p /(p+\nu+1)}\left(\frac{1}{\psi^{2 p /(p+\nu+1)-1}(r)}-\frac{1}{\psi^{2 p /(p+\nu+1)-1}\left(R_{k}\right)}\right) \\
& \geq C \int_{R_{k}}^{r} e^{(n-1) A s+\zeta s} d s .
\end{aligned}
$$

Since $\psi(r) \rightarrow \infty$ as $r \rightarrow \infty$, from (52) we get a contradiction to (55) as $r \rightarrow \infty$. That is, the case (ii) is impossible.

Remark 5. The actual estimates about the volume element that we needed in the proof of Theorem 2 are (b) in Remark 2 and

(d) there is a function $\chi(r), \chi(r) \rightarrow 0$ as $r \rightarrow \infty$, and $r_{1}>0$ such that

$$
\frac{\partial \log \sqrt{g_{\theta}}}{\partial r} \geq(n-1) B+\chi(r) \text { for } r>r_{1} ;
$$

(e) there are positive constants $C, r_{1}$ and a function $\eta(r), \eta(r) \rightarrow 0$ as $r \rightarrow \infty$, such that

$$
\sqrt{g_{\theta}} \leq C e^{(n-1) A r+\eta(r) r} \text { for } r>r_{1} .
$$

Remark 6. All arguments in the proofs of Theorems $1, \mathrm{~A}$, and 2 are near $\infty$. Therefore if near $\infty$ on $\left(M^{n}, g\right)$ we have the estimates in Remarks 2 and 5, the conclusions of these theorems hold. For example, we can apply our results to the manifold $\left(M_{1}^{n}, g_{2}\right)$, where $\left(M_{1}^{n} \backslash E_{1}, g_{2}\right)=\left(M^{n} \backslash E_{0}, g\right), E_{0}$ is a compact set in $M^{n}, E_{1}$ is a subset in $M_{1}^{n}$, and $\left(M^{n}, g\right)$ is a manifold satisfying our basic assumptions.

Remark 7. One also observes that there might exist positive solutions to the equation in problem (P) satisfying (25), as shown in the following example.

Example 2. Let $\left(M^{n}, g\right)$ be a manifold satisfying our basic assumptions. Set $v=\phi(r) e^{w r}$, where $\phi$ is a cutoff function which vanishes near 0 and equals to 1 for $r>1$. Because $\partial \log \sqrt{g} / \partial r$ is bounded for $r>1$, we can choose $w<0,|w|$ large, such that

$$
w^{2}+\frac{\partial \log \sqrt{g}}{\partial r} w+|S|>0 \text { for } r \text { large. }
$$

Then for

$$
K(x)=\left(-\Delta_{g} v+S v\right) v^{-(n+2) /(n-2)}
$$


$v$ satisfies the corresponding equation, and $K$ satisfies $(25)$. But $v^{4 /(n-2)} g$ is not complete because $w<0$.

\section{EXISTENCE RESULTS}

In this section, under the basic assumptions on the manifold, we prove Theorem 4. Notice that there is a constant $r(k, \alpha)$ such that for $r>r(k, \alpha)$, $-e^{-\alpha r^{k}} \leq-r^{2 k} e^{-\alpha r^{k+1}}$. Then in order to prove Theorem 4 , we only have to prove the following result.

Theorem $5(n \geq 3)$. Given constants $A, B, r_{0}, k, \alpha>0$, there is a constant $C\left(A, B, n, r_{0}, k, \alpha\right)>0$, such that if

$$
\max _{M^{n}} K \leq C\left(A, B, n, r_{0}, k, \alpha\right),
$$

and for some positive constant $C_{2}$,

$$
-C_{2} \leq K(x) \leq-r(x)^{2(k-1)} e^{-\alpha r(x)^{k}} \quad \text { for } r(x) \geq r_{0} .
$$

Then problem (P) has at least one solution.

Using the inhomogeneity of the equation in problem $(\mathrm{P})$, we see that Theorem 5 is equivalent to

Theorem 5* $(n \geq 3)$. Given constants $A, B, r_{0}, k, \alpha>0$, there is a constant $C\left(A, B, n, r_{0}, k, \alpha\right)>0$ such that if for some constants $C_{1}, C_{2} \max _{M^{n}} K \leq$ $C_{1} C\left(A, B, n, r_{0}, k, \alpha\right)$, and

$$
-C_{2} \leq K(x) \leq-C_{1} r(x)^{2(k-1)} e^{-\alpha r(x)^{k}} \quad \text { for } r(x) \geq r_{0} .
$$

Then problem (P) has at least one solution.

Remark 8. Condition (56) is automatically satisfied if $\max _{M^{n}} K \leq 0$. Therefore we have

Theorem $3(n \geq 3)$. If $K \leq 0$ on $M^{n}$, and there are positive constants $C_{1}$, $C_{2}, \alpha, k, r_{0}$ such that $-C_{2} \leq K(x) \leq-C_{1} r(x)^{2(k-1)} e^{-\alpha r(x)^{k}}$ for $r(x) \geq r_{0}$. then problem (P) has at least one solution.

Remark 9. Compare Theorem 5 with Theorem 4 in [5]; we removed the restriction on the ratio of $A^{2} / B^{2}$. In Theorem 4 of [5], the authors assumed

$$
A^{2} / B^{2} \leq(n-1)^{2} / n(n-2) \text {. }
$$

Remark 10. Also, one should notice that Theorem 5 applys to the case that $K$ might approach 0 as $r \rightarrow \infty$. This case is not covered by Theorem 4 in [5].

Proof of Theorem 5. Choose a constant $\varrho$ such that

$$
\varrho=10^{7} n(n-2) \frac{A^{2}}{r_{0}}+\frac{1}{r_{0}}+1 .
$$

We multiply the metric $g$ by the constant $\varrho^{2}$. That is consider $g_{5}=\varrho^{2} g$. Since $g_{5}$ is conformal to $g$ by a constant multiple, we only have to solve problem (P) for the metric $g_{5}$. For convenience we denote $g_{5}$ by $g$. Then

$$
\operatorname{Ricc}(g) \geq-(n-1) \frac{A^{2}}{\varrho^{2}} ; \quad \operatorname{Sec}(g) \leq-\frac{B^{2}}{\varrho^{2}} \text {. }
$$


The condition about $K$ becomes

$$
-C_{2} \leq K(x) \leq-\left(\frac{r(x)}{\varrho}\right)^{2(k-1)} e^{-\alpha r(x)^{k} / \varrho^{k}} \text { for } r(x) \geq \varrho r_{0},
$$

since the distance is scaled by the constant $\varrho$.

Without loss of generality, we may assume $k>2$. Let

$$
m_{1}=\max _{M^{n}} K .
$$

We first consider $m_{1}>0$. By multiplying the solution by a constant, using the inhomogeneity of the equation, we only have to solve the following PDE problem:

$$
\begin{aligned}
& -\Delta_{g} u+S u=\left(K / m_{1}\right) u^{(n+2) /(n-2)} \text { on } M^{n} \\
& u^{4 /(n-2)} g \text { is complete on } M^{n} ; \\
& u>0 \text { on } M^{n} .
\end{aligned}
$$

We will solve problem $\left(\mathrm{P}^{*}\right)$ by constructing a pair of super- and sub-solutions.

Choose a smooth function $\phi(r)$ on $(0, \infty)$ as follows:

(1) $\phi(r) \geq 0$ on $(0, \infty)$;

(2) $\phi^{\prime}(r) \geq 0$ on $(0, \infty)$;

(3) $\phi(r)=0$ on $\left(0, \varrho r_{0}\right)$;

(4) $\phi(r)=1$ on $\left(3 \varrho r_{0}, \infty\right)$;

(5) $\phi\left(2 \varrho r_{0}\right)=\varepsilon_{0}$;

(6) $\left|\phi^{\prime}(r)\right| \leq C\left(\varrho r_{0}\right)^{-1} \varepsilon_{0},\left|\phi^{\prime \prime}(r)\right| \leq C\left(\varrho r_{0}\right)^{-2} \varepsilon_{0}$ on $\left(\varrho r_{0}, 2 \varrho r_{0}\right)$.

The constant $0<\varepsilon_{0}<1$ will be specified later.

(7) $\left|\phi^{\prime}(r)\right| \leq C\left(\varrho r_{0}\right)^{-1},\left|\phi^{\prime \prime}(r)\right| \leq C\left(\varrho r_{0}\right)^{-2}$ on $\left(2 \varrho r_{0}, 3 \varrho r_{0}\right)$.

The super solution we will look for is of the form

$$
v=v_{1} \phi+(1-\phi) v_{2}
$$

where $v_{1}=e^{w_{1} r^{k}}, v_{2}=z_{2} e^{w_{2} r} f(r)$. The constants $z_{2}, w_{1}, w_{2}$, and the function $f(r)$ will be specified later.

Let

$$
L v=\left(-\Delta_{g} v+S v\right) v^{-(n+2) /(n-2)} .
$$

We want to compute $L v$ on four different intervals.

(1) $0 \leq r \leq \varrho r_{0}$,

(2) $r \geq 3 \varrho r_{0}$

(3) $\varrho r_{0} \leq r \leq 2 \varrho r_{0}$

(4) $2 \varrho r_{0} \leq r \leq 3 \varrho r_{0}$.

(1) On $\left(0, \varrho r_{0}\right)$. Notice that if $v=v(r)$, a function only depending on $r$, we have

$$
\Delta_{g} v=\frac{\partial^{2} v}{\partial r^{2}}+\frac{\partial \log \sqrt{g}}{\partial r} \frac{\partial v}{\partial r}
$$

Then it is easy to verify

$$
\begin{aligned}
L v_{2}=z_{2}^{-\frac{4}{(n-2)}} e^{-\frac{4}{(n-2)} w_{2} r}\{ & -\left(w_{2}^{2} f(r)+2 w_{2} f^{\prime}(r)+f^{\prime \prime}(r)\right) \\
& \left.-\frac{\partial \log \sqrt{g}}{\partial r}\left(w_{2} f(r)+f^{\prime}(r)\right)+S f(r)\right\} f^{-\frac{(n+2)}{(n-2)}} .
\end{aligned}
$$


Set

where $\mu, \xi$ will be specified later.

$$
f(r)=\left(1+\frac{\sinh \mu r}{\cosh \mu r}\right)^{\xi}
$$

A computation shows

$$
\begin{aligned}
w_{2}^{2} f(r)+2 w_{2} f^{\prime}(r)+f^{\prime \prime}(r)+\frac{\partial \log \sqrt{g}}{\partial r}\left(w_{2} f(r)+f^{\prime}(r)\right)+|S| f(r) \\
=\left(1+\frac{\sinh \mu r}{\cosh \mu r}\right)^{\xi-2}\left\{w_{2}^{2}\left(1+\frac{\sinh \mu r}{\cosh \mu r}\right)^{2}+2 \mu \xi w_{2}\left(1+\frac{\sinh \mu r}{\cosh \mu r}\right) \frac{1}{(\cosh \mu r)^{2}}\right. \\
+\mu^{2} \xi(\xi-1) \frac{1}{(\cosh \mu r)^{4}}-2 \mu^{2} \xi\left(1+\frac{\sinh \mu r}{\cosh \mu r}\right) \frac{\sinh \mu r}{(\cosh \mu r)^{3}} \\
+\frac{\partial \log \sqrt{g}}{\partial r} w_{2}\left(1+\frac{\sinh \mu r}{\cosh \mu r}\right)^{2} \\
+\mu \xi\left(1+\frac{\sinh \mu r}{\cosh \mu r}\right) \frac{1}{(\cosh \mu r)^{2}} \frac{\partial \log \sqrt{g}}{\partial r} \\
\left.+|S|\left(1+\frac{\sinh \mu r}{\cosh \mu r}\right)^{2}\right\} .
\end{aligned}
$$

Define

$$
t=\frac{\sinh \mu r}{\cosh \mu r} \text { for } r \geq 0 .
$$

Using $(\cosh \mu r)^{2}-(\sinh \mu r)^{2}=1$, we have

$$
\begin{array}{r}
w_{2}^{2} f(r)+2 w_{2} f^{\prime}(r)+f^{\prime \prime}(r)+\frac{\partial \log \sqrt{g}}{\partial r}\left(w_{2} f(r)+f^{\prime}(r)\right)+|S| f(r) \\
=(1+t)^{\xi-2}\left\{\left(\begin{array}{r}
\left.w_{2}^{2}+\frac{\partial \log \sqrt{g}}{\partial r} w_{2}+|S|\right)(1+t)^{2}+2 \mu \xi w_{2}(1+t)\left(1-t^{2}\right) \\
+\mu^{2} \xi(\xi-1)\left(1-t^{2}\right)^{2}-2 \mu^{2} \xi(1+t)\left(1-t^{2}\right) t \\
\left.+\mu \xi(1+t)\left(1-t^{2}\right) \frac{\partial \log \sqrt{g}}{\partial r}\right\} \\
=(1+t)^{\xi}\left\{w_{2}^{2}+\frac{\partial \log \sqrt{g}}{\partial r} w_{2}+|S|+2 \mu \xi w_{2}(1-t)+\mu^{2} \xi(\xi-1)(1-t)^{2}\right. \\
\left.-2 \mu^{2} \xi(1-t) t+\mu \xi(1-t) \frac{\partial \log \sqrt{g}}{\partial r}\right\} .
\end{array}\right.\right.
\end{array}
$$

By (58),

$$
\frac{\partial \log \sqrt{g}}{\partial r}>0 \text { for } r>0
$$

Now in (63), we choose $\mu<0, \xi=\frac{1}{2}$, and $w_{2}=-\mu^{2}$ (the value of $\mu$ will be specified in a moment). We have

$$
\begin{array}{r}
w_{2}^{2} f(r)+2 w_{2} f^{\prime}(r)+f^{\prime \prime}(r)+\frac{\partial \log \sqrt{g}}{\partial r}\left(w_{2} f(r)+f^{\prime}(r)\right)+|S| f(r) \\
\leq(1+t)^{1 / 2}\left\{\mu^{4}+|S|-\mu^{3}(1-t)-\frac{1}{4} \mu^{2}(1-t)^{2}-\mu^{2}(1-t) t\right\} .
\end{array}
$$

Here we used the fact that for $\mu<0, r \geq 0,-1<t<0$. Furthermore, for $0<r<3 \varrho r_{0}$,

$$
|t|=\left|\frac{\sinh \mu r}{\cosh \mu r}\right|=\frac{1-e^{2 \mu r}}{1+e^{2 \mu r}} \leq 4 \mu^{2} r \leq 12 \mu^{2} \varrho r_{0}
$$


Then (65) implies (also use the fact that $-1<t \leq 0$.)

$$
\begin{aligned}
w_{2}^{2} f(r) & +2 w_{2} f^{\prime}(r)+f^{\prime \prime}(r)+\frac{\partial \log \sqrt{g}}{\partial r}\left(w_{2} f(r)+f^{\prime}(r)\right)+|S| f(r) \\
& \leq(1+t)^{1 / 2}\left\{\mu^{4}+|S|+2|\mu|^{3}-\frac{1}{4} \mu^{2}+2 \mu^{2}|t|\right\} \\
& =(1+t)^{1 / 2}\left\{|S|+\mu^{2}\left(-\frac{1}{4}+2|t|+\mu^{2}+2|\mu|\right)\right\} \\
& \leq(1+t)^{1 / 2}\left\{|S|+\mu^{2}\left(-\frac{1}{4}+24 \mu^{2} \varrho r_{0}+\mu^{2}+2|\mu|\right)\right\} .
\end{aligned}
$$

By $(58)$,

$$
|S| \leq \frac{n(n-2)}{4} \frac{A^{2}}{\left(\varrho r_{0}\right)^{2}} .
$$

Now in (66), choose $\mu=-1 / \sqrt{768 \varrho r_{0}}$. Then from (66) and (57), we have (68)

$$
\begin{aligned}
& w_{2}^{2} f(r)+2 w_{2} f^{\prime}(r)+f^{\prime \prime}(r)+\frac{\partial \log \sqrt{g}}{\partial r}\left(w_{2} f(r)+f^{\prime}(r)\right)+|S| f(r) \\
& \leq(1+t)^{1 / 2}\left\{\frac{1}{768 \varrho r_{0}}\left(-\frac{1}{4}+\frac{24}{768}+\frac{1}{768 \varrho r_{0}}+\frac{2}{\sqrt{768 \varrho r_{0}}}\right)+\frac{n(n-2)}{4} \frac{A^{2}}{\left(\varrho r_{0}\right)^{2}}\right\} \\
& \quad \leq(1+t)^{1 / 2}\left\{\frac{1}{768 \varrho r_{0}}\left(-\frac{1}{4}+\frac{24}{768}+\frac{1}{768}+\frac{2}{\sqrt{768}}\right)+\frac{n(n-2)}{4} \frac{A^{2}}{\left(\varrho r_{0}\right)^{2}}\right\} \\
& \leq(1+t)^{1 / 2}\left\{-\frac{1}{6144 \varrho r_{0}}+\frac{n(n-2)}{4} \frac{A^{2}}{\left(\varrho r_{0}\right)^{2}}\right\}<0 .
\end{aligned}
$$

Here we also used a consequence of (57) that $\varrho r_{0}>1$.

From (62) and (68), we can choose $z_{2}=z_{2}\left(A, n, r_{0}\right)>0$ small enough such that

$$
L v_{2} \geq 1 \text { on }\left(0,3 \varrho r_{0}\right)
$$

Therefore

$$
L v_{2} \geq K / m_{1} \quad \text { on }\left(0, \varrho r_{0}\right) \text {. }
$$

(2) On $\left(3 \varrho r_{0}, \infty\right)$. We compute

$$
L v_{1}=-e^{-4 w_{1} r^{k} /(n-2)}\left(k^{2} w_{1}^{2} r^{2(k-1)}+k(k-1) w_{1} r^{k-2}+\frac{\partial \log \sqrt{g}}{\partial r} k w_{1} r^{k-1}+|S|\right) \text {. }
$$

Choose $w_{1}=\frac{1}{4}(n-2) \alpha / \varrho^{k}$. By (4),

$$
\begin{aligned}
\frac{\partial \log \sqrt{g}}{\partial r} & \leq(n-1)\left(\frac{A}{\varrho}\right) \frac{\cosh (A r / \varrho)}{\sinh (A r / \varrho)} \\
& \leq(n-1)\left(\frac{A}{\varrho}\right) \frac{1+e^{-6 A r_{0}}}{1-e^{-6 A r_{0}}} \quad \text { for } r \geq 3 \varrho r_{0}
\end{aligned}
$$

Then

$$
\begin{gathered}
k^{2} w_{1}^{2} r^{2(k-1)}+k(k-1) w_{1} r^{k-2}+\frac{\partial \log \sqrt{g}}{\partial r} k w_{1} r^{k-1}+|S| \\
\leq r^{2(k-1)} C_{3}\left(n, A, r_{0}, k, \alpha\right) \text { for } r \geq 3 \varrho r_{0} .
\end{gathered}
$$


Assume

$$
1 / m_{1} \geq C_{3}\left(n, A, r_{0}, k, \alpha\right) \varrho^{2(k-1)}
$$

(later we will choose the constant $C\left(A, B, n, r_{0}, k, \alpha\right)$ in the assumption of Theorem 5 such that (73) is true). Then we have

$$
L v_{1} \geq-\frac{1}{m_{1}}\left(\frac{r}{\varrho}\right)^{2(k-1)} e^{-\alpha r^{k} / \varrho^{k}} \geq \frac{K}{m_{1}} \quad \text { for } r \geq 3 \varrho r_{0} .
$$

(3) On $\left(\varrho r_{0}, 2 \varrho r_{0}\right)$. By a computation, we have

$$
\begin{aligned}
& L v=(v)^{-\frac{n+2}{n-2}}\left\{\phi v_{1}^{\frac{n+2}{n-2}} L v_{1}+(1-\phi) v_{2}^{\frac{n+2}{n-2}} L v_{2}\right. \\
&\left.\quad-\frac{\partial \log \sqrt{g}}{\partial r}\left(v_{1}-v_{2}\right) \phi^{\prime}(r)-\left(v_{1}-v_{2}\right) \phi^{\prime \prime}(r)-2 \phi^{\prime}\left(v_{1}^{\prime}-v_{2}^{\prime}\right)\right\} .
\end{aligned}
$$

From properties (1)-(6) of $\phi$, Proposition 1, and the construction of $v_{1}, v_{2}$, we see that there is a constant $C^{*}\left(n, A, B, r_{0}, k, \alpha\right)$ such that for $\varrho r_{0} \leq r \leq$ $2 \varrho r_{0}$,

$$
L v \geq(v)^{-(n+2) /(n-2)}\left\{-\varepsilon_{0} C^{*}\left(n, A, B, r_{0}, k, \alpha\right)+\left(1-\varepsilon_{0}\right) v_{2}^{(n+2) /(n-2)} L v_{2}\right\} .
$$

Using (69) gives

$$
L v \geq(v)^{-(n+2) /(n-2)}\left\{-\varepsilon_{0} C^{*}\left(n, A, B, r_{0}, k, \alpha\right)+\left(1-\varepsilon_{0}\right) v_{2}^{(n+2) /(n-2)}\right\} .
$$

Now we see that there is a small $\varepsilon_{0}=\varepsilon_{0}\left(n, A, B, r_{0}, k, \alpha\right)>0$ such that if $\phi$ is defined by this $\varepsilon_{0}$, we have

$$
L v \geq 0 \text { on }\left(\varrho r_{0}, 2 \varrho r_{0}\right) \text {. }
$$

Since $K \leq 0$ on $\left(\varrho r_{0}, 2 \varrho r_{0}\right)$,

$$
L v \geq K / m_{1} \text { on }\left(\varrho r_{0}, 2 \varrho r_{0}\right) \text {. }
$$

(4) On $\left(2 \varrho r_{0}, 3 \varrho r_{0}\right)$. By (69) and (75), we have that on $\left(2 \varrho r_{0}, 3 \varrho r_{0}\right)$,

$$
\begin{aligned}
L v \geq(v)^{-(n+2) /(n-2)}\left\{\phi v_{1}^{(n+2) /(n-2)} L v_{1}\right. & -\frac{\partial \log \sqrt{g}}{\partial r}\left(v_{1}-v_{2}\right) \phi^{\prime}(r) \\
& \left.-\left(v_{1}-v_{2}\right) \phi^{\prime \prime}(r)-2 \phi^{\prime}\left(v_{1}^{\prime}-v_{2}^{\prime}\right)\right\} .
\end{aligned}
$$

Now if at $r_{1}$ with $2 \varrho r_{0} \leq r_{1} \leq 3 \varrho r_{0}$,

$$
\begin{aligned}
& \phi v_{1}^{(n+2) /(n-2)} L v_{1}\left(r_{1}\right)-\frac{\partial \log \sqrt{g}}{\partial r}\left(v_{1}-v_{2}\right) \phi^{\prime}\left(r_{1}\right) \\
& -\left(v_{1}-v_{2}\right) \phi^{\prime \prime}\left(r_{1}\right)-2 \phi^{\prime}\left(r_{1}\right)\left(v_{1}^{\prime}\left(r_{1}\right)-v_{2}^{\prime}\left(r_{1}\right)\right) \geq 0,
\end{aligned}
$$

we certainly have

$$
L v\left(r_{1}\right) \geq 0
$$

If at $r_{1}$ with $2 \varrho r_{0} \leq r_{1} \leq 3 \varrho r_{0}$,

$$
\begin{aligned}
& \phi v_{1}^{(n+2) /(n-2)} L v_{1}\left(r_{1}\right)-\frac{\partial \log \sqrt{g}}{\partial r}\left(v_{1}-v_{2}\right) \phi^{\prime}\left(r_{1}\right) \\
& -\left(v_{1}-v_{2}\right) \phi^{\prime \prime}\left(r_{1}\right)-2 \phi^{\prime}\left(r_{1}\right)\left(v_{1}^{\prime}\left(r_{1}\right)-v_{2}^{\prime}\left(r_{1}\right)\right) \leq 0,
\end{aligned}
$$


and noting that on $\left(2 \varrho r_{0}, 3 \varrho r_{0}\right), \phi \geq \varepsilon_{0}$, then $v \geq \varepsilon_{0} v_{1}$ on $\left(2 \varrho r_{0}, 3 \varrho r_{0}\right)$, we have

(80)

$$
\begin{aligned}
L v\left(r_{1}\right) \geq\left(\varepsilon_{0} v_{1}\right)^{-(n+2) /(n-2)}\{ & \phi v_{1}^{(n+2) /(n-2)} L v_{1}\left(r_{1}\right) \\
& -\frac{\partial \log \sqrt{g}}{\partial r}\left(v_{1}\left(r_{1}\right)-v_{2}\left(r_{1}\right)\right) \phi^{\prime}\left(r_{1}\right) \\
& \left.-\left(v_{1}-v_{2}\right) \phi^{\prime \prime}\left(r_{1}\right)-2 \phi^{\prime}\left(r_{1}\right)\left(v_{1}^{\prime}\left(r_{1}\right)-v_{2}^{\prime}\left(r_{1}\right)\right)\right\} .
\end{aligned}
$$

Hence in all cases there is a constant $C_{4}\left(n, A, B, r_{0}, k, \alpha\right)$ such that

$$
L v \geq-C_{4}\left(n, A, B, r_{0}, k, \alpha\right) \text { on }\left(2 \varrho r_{0}, 3 \varrho r_{0}\right) \text {. }
$$

Then in order to have

$$
L v \geq K / m_{1} \text { on }\left(2 \varrho r_{0}, 3 \varrho r_{0}\right),
$$

we only need that on $\left(2 \varrho r_{0}, 3 \varrho r_{0}\right)$,

$$
-\frac{1}{m_{1}}\left(\frac{r}{\varrho}\right)^{2(k-1)} e^{-\alpha r^{k} / \varrho^{k}} \leq-C_{4}\left(n, A, B, r_{0}, k, \alpha\right) .
$$

It is enough to have

$$
\frac{1}{m_{1}} \geq \frac{1}{\left(2 r_{0}\right)^{2(k-1)}} e^{\alpha\left(3 r_{0}\right)^{k}} C_{4}\left(n, A, B, r_{0}, k, \alpha\right) .
$$

Now it is clear that if we choose the constant $C\left(A, B, n, r_{0}, k, \alpha\right)$ in the assumption of Theorem 5 in such a way that

$$
\begin{aligned}
& C^{-1}\left(A, B, n, r_{0}, k, \alpha\right) \\
& =\max \left\{\frac{1}{\left(2 r_{0}\right)^{2(k-1)}} e^{\alpha\left(3 r_{0}\right)^{k}} C_{4}\left(n, A, B, r_{0}, k, \alpha\right),\right. \\
& \left.C_{3}\left(n, A, r_{0}, k, \alpha\right) \varrho^{2(k-1)}\right\} .
\end{aligned}
$$

Then (73) and (83) are true. Therefore (82) is true. Now (70), (74), (77), and (82) imply that $v$ is a supersolution to the equation in problem $\left(\mathrm{P}^{*}\right)$.

On the other hand, since $w_{1}>0$, we can choose a small constant $z_{3}>0$ such that $z_{3} \leq v$. But when $z_{3}$ is small, we have

$$
L z_{3}=z_{3}^{-4 /(n-2)} S \leq K / m_{1} \text { on } M^{n}
$$

by the assumption on $K$ and the fact that $S \leq C\left(n, A, r_{0}\right)<0$. Therefore for $z_{3}$ small, $z_{3}$ is a subsolution to the equation in problem $\left(\mathrm{P}^{*}\right)$.

Now Proposition 2 yields a solution $u$ to the equation in problem $\left(\mathrm{P}^{*}\right)$ satisfying $z_{3} \leq u \leq v$. It is clear that this solution is positive and also makes $u^{4 /(n-2)} g$ complete because $g$ is complete and $u \geq z_{3}$.

In the case that $\max K \leq 0$, we choose a positive constant $m_{1}$ such that $1 / m_{1}>C^{-1}\left(n, A, B, r_{0}, k, \alpha\right)$. Then we can proceed as in the the previous case to conclude the existence of a solution of problem $\left(\mathrm{P}^{*}\right)$. Therefore there is no restriction for $\max _{M^{n}} K$ when $K \leq 0$. 
Remark 11. From the proof of Theorem 5, one can check that the estimates needed for the volume element are (64), (72), and

$$
\frac{\partial \log \sqrt{g}}{\partial r}<C\left(r_{1}, r_{2}\right) \text { for } 0<r_{1}<r<r_{2} .
$$

Thus Theorem 5 actually holds for the following type of complete, noncompact, simply connected manifolds $\left(M^{n}, g\right)$ :

(1) $S_{0}<-C<0$;

(2) $\operatorname{Ricc}(g) \geq-(n-1) A^{2}, \operatorname{Sec}(g) \leq 0$.

Remark 12. Condition (56) is not a best possible condition in the following sense.

Claim. Let $\left(M^{n}, g\right)$ be a manifold satisfying our basic assumptions. Given positive constants $A, B, r_{0}, k, \alpha, C_{2}$; for any $m>0$, there is a function $K_{m}$ on $\left(M^{n}, g\right)$ such that

$$
-C_{2} \leq K_{m}(x) \leq-r(x)^{2(k-1)} e^{-\alpha r(x)^{k}} \quad \text { for } r(x) \geq r_{0} .
$$

But $\max _{M^{n}} K_{m} \geq m$, and problem (P) is solvable for this $K_{m}$.

Sketch of the construction. Choose a function $K$ on $M^{n}$ such that

$$
-C_{2} \leq K(x) \leq-r(x)^{2(k-1)} e^{-\alpha r(x)^{k}} \text { for } r(x) \geq r_{0} .
$$

By (4),

$$
\frac{\partial \log \sqrt{g}}{\partial r} \geq C(B, n) \frac{1}{r} \text { for } 0<r<1 .
$$

Also notice that (65) actually holds as

$$
\begin{aligned}
w_{2}^{2} f(r) & +2 w_{2} f^{\prime}(r)+f^{\prime \prime}(r)+\frac{\partial \log \sqrt{g}}{\partial r}\left(w_{2} f(r)+f^{\prime}(r)\right)+|S| f(r) \\
\leq & (1+t)^{1 / 2}\left\{\mu^{4}+|S|-\mu^{3}(1-t)-\frac{1}{4} \mu^{2}(1-t)^{2}-\mu^{2}(1-t) t\right\} \\
& -\mu^{2} \frac{\partial \log \sqrt{g}}{\partial r}(1+t)^{1 / 2} .
\end{aligned}
$$

Now replace $m_{1}$ in the definition of $(60)$ by

$$
m_{1}=\max _{r(x) \geq 1} K(x) .
$$

Then for the same choice of $\mu, z_{2}, w_{2}, \xi$, we see that we can modify $K$ on $\{x \mid r(x) \leq 1\}$ to get a $K_{m}$ satisfying the requirement in the Claim and problem (P) is solvable for this $K_{m}$ by the same proof given in the proof of Theorem 5.

Remark 13. From Remark 12, one might guess that probably no restriction on $\max K$ is necessary. In the following, we give an example to show that the situation might be complicated.

Example 3. Consider the hyperbolic space form $H^{n}(-1)$. Let

$$
v_{1}=\left(\frac{1}{4(\cosh r / 2)^{2}}\right)^{(n-2) / 2},
$$


Then it is easy to see that $-\Delta_{g} v_{1}+S v_{1}=0$. Also set $v_{2}=e^{w r}$ with $w<$ $-(n+2) / 2$ and

$$
w^{2}+w \frac{\partial \log \sqrt{g}}{\partial r}+|S| \geq C>0 \quad \text { for } r \geq r_{1}
$$

where $r_{1}$ is some number. Such $w$ exists because $\partial \log \sqrt{g} / \partial r$ is bounded for $r>1$.

Now consider the metric $g_{6}=\left(v_{1}+v_{2}\right)^{4 /(n-2)} g$. It is clear that $g_{6}$ is not complete because $w<0$. Furthermore the scalar curvature of $g_{6}$ is

$$
\begin{aligned}
K & =\gamma_{n}\left(v_{1}+v_{2}\right)^{-(n+2) /(n-2)}\left(-\Delta_{g}\left(v_{1}+v_{2}\right)+S\left(v_{1}+v_{2}\right)\right) \\
& =-\gamma_{n}\left(v_{1}+v_{2}\right)^{-(n+2) /(n-2)}\left(w^{2}+w \frac{\partial \log \sqrt{g}}{\partial r}+|S|\right) e^{w r} \\
& \approx-C e^{(w+(n+2) / 2) r} \quad \text { as } r \rightarrow \infty .
\end{aligned}
$$

Therefore for some $C_{1}, C_{2}, r_{0}, K$ satisfies

$$
-C_{2} \leq K(x) / C_{1} \leq-e^{(w+(n+2) / 2) r(x)} \quad \text { for } r(x)>r_{0} .
$$

That is, we demonstrated a function $K / C_{1}$ which satisfies the assumptions of Theorem 5 except $(56)$, but the corresponding equation in problem $(\mathrm{P})$ has a positive solution $u=v_{1}+v_{2}$ which does not make $u^{4 /(n-2)} g$ complete.

\section{REFERENCES}

1. L. V. Ahlfors, An extension of Schwarz's lemma, Trans. Amer. Math. Soc. 43 (1938), 359364.

2. T. Aubin, Equations differentielles non lineaires et probleme de Yamabe concernant la courbure scalaire, J. Math. Pures Appl. 55 (1976), 269-296.

3. __ Nonlinear analysis on manifolds, Springer, New York, 1982.

4. P. Aviles, Conformal complete metrics with prescribed non-negative Gaussian curvature in $R^{2}$, Invent. Math. 83 (1986), 519-544.

5. P. Aviles and R. McOwen, Conformal deformations of complete manifolds with negative curvature, J. Differential Geom. 21 (1985), 269-281.

6. Conformal deformation to constant negative scalar curvature on noncompact Riemannian manifolds, J. Differential Geom. 27 (1988), 225-239.

7. J. Bland and M. Kalka, Complete metrics conformal to the hyperbolic disc, Proc. Amer. Math. Soc. 97 (1986), 128-132.

8. E. Calabi, An extension of E. Hopf's maximum principle with an application to Riemannian geometry, Duke Math. J. 25 (1958), 45-56.

9. N. Garofalo and F. H. Lin, Unique continuation for elliptic operators: A geometric-variational approach, Comm. Pure Appl. Math. 40 (1987), 347-366.

10. D. Gilbarg and N. S. Trüdinger, Elliptic partial differential equations of second order, Springer, Berlin, 1977.

11. R. Greene and H. Wu, Function theory on manifolds which possess a pole, Lecture Notes in Math., vol. 699, Springer-Verlag, and New York, 1979.

12. Z. Jin, A counter-example to the Yamabe problem for complete noncompact manifolds, Lecture Notes in Math., vol. 1306, Springer-Verlag, Berlin and New York, 1988, pp. 93-101.

13. J. Kazdan, Prescribing the curvature of a Riemannian manifold, CBMS Regional Conf. Ser. in Math., no. 51, Amer. Math. Soc., Providence, R.I., 1985.

14. _ـ Unique continuation in geometry, Comm. Pure Appl. Math. 41 (1988), 667-681.

15. J. Lee and T. Parker, The Yamabe problem, Bull. Amer. Math. Soc. 17 (1987), 37-91. 
16. R. McOwen, Conformal metrics in $R^{2}$ with prescribed Gaussian curvature and positive total curvature, Indiana Univ. Math. J. 34 (1985), 97-104.

17. W. M. Ni, On the elliptic equation $\Delta u+K(x) u^{(n+2) /(n-2)}=0$, its generalizations, and applications in geometry, Indiana Univ. Math. J. 31 (1982), 493-529.

18. Gaussian curvatures, Invent. Math. 66 (1982), 343-352.

19. R. Osserman, On the inequality $\Delta u \geq f(u)$, Pacific J. Math. 7 (1957), 1641-1647.

20. D. H. Sattinger, Conformal metrics on $R^{2}$ with prescribed curvatures, Indiana Univ. Math. J. 22 (1972), 1-4.

21. R. Schoen, Conformal deformation of a Riemannian metric to constant scalar curvature, J. Differential Geom. 20 (1984), 479-495. , A report on some recent progress on nonlinear problems in geometry, preprint.

23. R. Schoen and S. T. Yau, Conformally flat manifolds, Kleinian groups and scalar curvature, Invent. Math. 92 (1988), 47-71.

24. G. Stampacchia, On some regular multiple integral problems in the calculus of variations, Comm. Pure Appl. Math. 16 (1963), 383-421.

25. N. Trüdinger, Remarks concerning the conformal deformation of Riemannian structures on compact manifolds, Ann. Scuola Norm. Sup. Pisa 22 (1968), 265-274.

26. H. Yamabe, On the deformation of Riemannian structures on compact manifolds, Osaka Math. J. 12 (1960), 21-37.

Department of Mathematics, University of Michigan, AnN Arbor, Michigan 48109

E-mail address: zhiren.jin@math. Isa.umich.edu 